\section{Darwin and disease}

\author{
Robin A. Weiss
}

Evolution of Infectious Disease. By Paul W. Ewald. Oxford University Press: 1994. Pp. 298. \$35, £27.50.

The Evolutionary Biology of Viruses. Edited by Stephen S. Morse. Raven: 1994. Pp. 353. $\$ 87$.

I HAVE not picked up a book on infectious disease with so much anticipation as Paul Ewald's Evolution of Infectious Disease since reading William McNeil's Plagues and Peoples more than 15 years ago. I was not disappointed: Ewald's book is as teeming with ideas as some of us are with microbes. My problem, however, was to discern those ideas that are destined for transmission and further evolution from those that resemble defective interfering notions. Ewald's mission is to persuade us to view infectious disease in evolutionary terms, an approach he calls 'Darwinian medicine'. His main thesis is that the virulence of infectious microorganisms will vary according to natural selection acting on factors favouring their transmission. He reminds us that an agent of infectious disease can quickly replicate in a new host to give upwards of $10^{10}$ infectious particles, with opportunities for rapid evolution and change in virulence, for example by becoming resistant to antibiotics and drugs. Whereas evolutionists have accepted for a decade or more that infectious organisms can increase or decrease their virulence in the host according to selective pressures, the rest of us still tend to mouth the old saw that the longer the parasite and host live together, the further their relationship will evolve towards benign commensality. Ewald shatters this misconception. He illustrates how "cultural vectors" can predispose to increasing virulence, whether it be diarrhoea from cholera or AIDS from HIV. He goes on to argue that by deliberately manipulating the dynamics of transmission we could reduce the severity of disease, and he makes a plea for more Darwinian thinking in medicine, epidemiology and public health.

\section{Models}

Evolution of Infectious Disease is a challenging and readable introduction to current thinking on the topic. As an experimental, laboratory-based virologist, I certainly found it stimulating. Ewald has plenty of pithy aphorisms: he says, for example, that from a pathogen's viewpoint "a dead host and an immune host are not much different". His book is easier going than the excellent modern classic by Robert M. May and Roy M. Anderson, Infectious Diseases of Humans: Dynamics and Control (Oxford University Press,
1991). But specialists in the field may find it irritating, for it lacks a certain rigour that one might expect in evolutionary theory and is devoid of any mathematical treatment to explore the robustness of the author's models of disease. Further, the closer Ewald approaches my own field of expertise, the riskier I find his premises and the more suspect his conclusions. This is largely on account of his ignorance of pathology on the one hand, and of molecular biology on the other. I do not think that chronic, persistent infections with, say, the hepatitis- $\mathrm{C}$ virus or the human immunodeficiency virus (HIV) can be treated in quite the same way as acute diseases, for which the virulence to the host is more likely to relate directly to their early transmission. For instance, I am not convinced, as Ewald is, that the virulence of human T-cell leukaemia virus type $I$ is bound to increase on account of blood transmission among intravenous drug abusers. And, if it does, would one expect tropical spastic paraparesis to outpace adult T-cell leukaemia because it is more commonly related to parenteral (blood) acquisition?

\section{Mechanisms}

Relating virulence to an understanding of pathogenesis would seen to be particularly important in AIDS, yet I was left frustrated by Ewald's chapters on this disease. His account of Kaposi's sarcoma is garbled; he fails to mention the high relative incidence of this sarcoma in other immunosuppressive conditions as seen, for example, in recipients of organ transplants. As to HIV itself, I would have liked to read an evolutionary discussion about the pathogenesis of this newly emerged virus. There are three major views of HIV pathogenesis: that it is an immunologically driven disease, akin to autoimmunity; that virulent substrains emerge in infected individuals; and that the overall diversity of HIV in vivo, rather than particular virulent substrains, eventually overcomes the repertoire of T-cell responses. Overall, I am not persuaded that virulence to the host relates solely to the dynamics of transmission of the parasite; some knowledge of the mechanism of pathogenesis is needed.

To be fair, Ewald does outline early in his book that some pathological effects of infection may be occasional, dead-end outcomes so far as the invading parasite is concerned. What he does not admit is that many of these 'side-effects', which do not influence the probability of transmission, are nonetheless major targets for improving human health. Take poliomyelitis as an example. The race in the $1950 \mathrm{~s}$ to develop a safe, efficacious polio vaccine and the current campaign of the World Health Organisation to eradicate polio virus (successfully achieved for the Americas) is driven by such a side-effect, namely the invasion of the central nervous system, in a minority of infected children. The virus is naturally transmitted by way of the gut and the immobilization of the host through the destruction of motor neurons would not appear to aid transmission. Notwithstanding Ewald' s sensible plea for Darwinian medicine, I hold that a significant proportion of mankind's morbidity and mortality, whether through poliomyelitis, cerebral malaria, elephantiasis or 20 per cent of cancers, results from pathologies that are not directly related to the transmission of the causative agent.

\section{Molecular details}

The molecular biology lacking in Evolution of Infectious Disease is all to be found in The Evolutionary Biology of Viruses. Stephen Morse has assembled a series of articles on evolution and on viral variation that represent much more than the usual set of reviews or conference proceedings. Most of the authors have written genuine essays on their subjects, probing the limits of current knowledge and ideas. Every chapter is informative. I benefited in particular from Morse's own introductory article, G. P. Garnett and R. Antia on the population biology of virus-host interactions, E. Domingo and J. J. Holland on mutation rates and rapid evolution of RNA viruses, and E. D. Kilbourne on host determination of viral evolution.

Morse points out that most discussions of viral evolution are generally outside the neo-Darwinian synthesis, and the relationship between viral variation and natural selection remains largely unexplored. In this volume, the processes generating genetic diversity (especially in RNA viruses lacking proof-reading during replication) is brought together with natural selection as a driving force in evolution. This synthesis is timely. A reviewer of a recent paper from my laboratory illustrating how immune escape of HIV can alter tropism (growth in macrophages) and vice versa, stated that there is no reason to think that the immune system exerts any selection on HIV in vivo, on the assumption that the generation of variants keeps the virus ahead of the immune system. Such referees should read this volume, in which that veteran evolutionist, Ernst Mayr, reminds us that evolution is a twostep process. The first step produces genetic variation, the second is the process of selection based on altered phenotype. The high mutation rates of many viruses has been noted, writes Mayr, but the individual progeny will later be subject to selection. Quite so.

Robin A. Weiss is in the Chester Beatty Laboratories, Institute of Cancer Research, 237 Fulham Road, London SW3 6JB, UK. 